\title{
Autophagy in the pathogen Candida albicans
}

\author{
Correspondence \\ Glen E. Palmer \\ gpalme@lsuhsc.edu
}

Received 22 August 2006

Revised 23 September 2006

Accepted 26 September 2006

\author{
Glen E. Palmer, Michelle N. Kelly and Joy E. Sturtevant
}

Department of MIP, Louisiana State University Health Sciences Center School of Dentistry, 1100 Florida Avenue, Box F8-130, New Orleans, LA 70119, USA

\begin{abstract}
Autophagy is a major cellular process that facilitates the bulk degradation of eukaryotic macromolecules and organelles, through degradation within the lysosomal/vacuole compartment. This has been demonstrated to influence a diverse array of eukaryotic cell functions including adaptation, differentiation and developmental programmes. For example, in Saccharomyces cerevisiae autophagy is required for sporulation and survival of nitrogen starvation. The opportunistic pathogen Candida albicans has the ability to colonize and cause disease within a diverse range of mammalian host sites. The ability to adapt and differentiate within the host is liable to be critical for host colonization and infection. Previous results indicated that the vacuole plays an important role in C. albicans adaptation to stress, differentiation, and survival within and injury of host cells. In this study the importance of vacuole-mediated degradation through the process of autophagy was investigated. This involved identification and deletion of ATG9, a C. albicans gene required for autophagy. The deletion strain was blocked in autophagy and the closely related cytoplasm to vacuole (cvt) trafficking pathway. This resulted in sensitivity to nitrogen starvation, but no defects in growth rate, vacuole morphology or resistance to other stresses. This indicates that the mutant has specific defects in autophagy/cvt trafficking. Given the importance of autophagy in the development and differentiation of other eukaryotes, it was surprising to find that the atg $9 \Delta$ mutant was unaffected in either yeast-hypha or chlamydospore differentiation. Furthermore, the atg $9 \Delta$ mutant survived within and killed a mouse macrophage-like cell line as efficiently as control strains. The data suggest that autophagy plays little or no role in C. albicans differentiation or during interaction with host cells.
\end{abstract}

\section{INTRODUCTION}

Autophagy is a major pathway by which eukaryotes are able to degrade cellular material. This has been shown to influence a wide array of biological phenomena including resistance to stress and starvation, cellular differentiation, development and ageing, programmed cell death, antigen presentation, clearance of intracellular pathogens, tumorigenesis and neurodegenerative disease (Mizushima, 2005). In the model yeast Saccharomyces cerevisiae, autophagy is critical for survival during nitrogen starvation, and to complete the differentiation process of sporulation (Takeshige et al., 1992; Tsukada \& Ohsumi, 1993). The molecular mechanism behind autophagy has been intensively studied in yeast and mammalian cells, and many of the components that are required have been identified (Abeliovich \& Klionsky, 2001; Klionsky, 2005). A portion of cytoplasmic material is bound in a double-membrane structure known as an autophagosome, which fuses with the vacuolar membrane to release a single-membrane-bound compartment within the vacuole lumen. Degradation of this

Abbreviations: AMS, $\alpha$-mannosidase; API, aminopeptidase I; CPY, carboxypeptidase $Y$; DIC, differential interference contrast; SAP, secreted aspartyl protease. material by the hydrolytic enzymes within the vacuole recycles cellular building blocks such as amino acids, which can be used in the synthesis of new macromolecules. Thus autophagy is typically considered a non-specific means to degrade 'bulk' cytoplasmic material. However, recent studies have revealed that under some conditions certain cargo is preferentially targeted for sequestration within the autophagosome (Nair \& Klionsky, 2005). For example, in $S$. cerevisiae under nutrient-replete conditions (i.e. when autophagy is repressed), the vacuolar hydrolases aminopeptidase I (API) and $\alpha$-mannosidase (AMS) are delivered from cytoplasm to vacuole via the cvt (cytoplasm to vacuole trafficking) pathway. The cvt pathway closely resembles autophagy, and involves sequestration of the cargo proteins within a double-membrane vesicle, which subsequently fuses to the vacuole to release its contents within the lumen. The vesicles formed are smaller than autophagosomes and appear to specifically deliver the API and AMS hydrolases. Interestingly, the cvt and autophagy pathways rely on much of the same cellular machinery, and many mutants defective in autophagy are also blocked in the cvt pathway (Abeliovich \& Klionsky, 2001; Thumm et al., 1994; Tsukada \& Ohsumi, 1993). Furthermore, under starvation conditions API and AMS are delivered to the vacuole in the 
autophagosome, underscoring the close relationship between these pathways.

Candida albicans is a commensal organism of some mammalian species including humans, where it commonly resides on mucosal surfaces. Under conditions of host immunosuppression C. albicans can invade host tissues to cause a diverse range of diseases. Infection of mucosal surfaces is common, with HIV patients being particularly susceptible to oral and oesophageal candidiasis (de Repentigny et al., 2004), while neutropenic patients are at risk of disseminated disease, with a high mortality rate (Maertens et al., 2001).

Given the importance of autophagy in a diverse array of eukaryotic cellular processes, we decided to establish the role of autophagy in C. albicans survival and differentiation within the host. To date the role the vacuole or indeed autophagy plays during Candida-host interaction has not been established. We hypothesized that autophagy is required for $C$. albicans adaptation and differentiation, two properties critical for survival within and infection of the mammalian host.

\section{METHODS}

Sequence analysis. C. albicans ATG9 and LAP4 homologues were identified through BLASTP searches on the C. albicans genome database (http://www.candidagenome.org/) using the S. cerevisiae protein sequences (http://www.yeastgenome.org/) as the query sequence. Protein sequences were aligned using the EMBOss pairwise align alogorithm (http://www.ebi.ac.uk/emboss/). Kyte-Doolittle hydropathy plots (Kyte \& Doolittle, 1982) were performed within the DS gene program (Accelrys).

Strain construction. Strains used in this study are described in Table 1. Gene deletion strains were constructed by the PCR-based approach described by Wilson et al. (1999), using the ura3 his1 arg4 strain BWP17, kindly provided by Dr A. Mitchell (Columbia University). $\operatorname{atg} 9 \Delta:: A R G 4$ and $\operatorname{atg} 9 \Delta::$ HIS1 deletion cassettes were amplified by PCR using pARG $\Delta$ Spe and pGEMHIS1 plasmids, respectively, as template with primers ATG9DISF and ATG9DISR (Table 2). BWP17 was first transformed with atg9D:: ARG4 to generate heterozygote strains BAA1 and BAA4. Each heterozygote strain was then transformed with the atg $9 \Delta::$ HIS1 cassette to generate the double deletion strains BAA1H1 and BAA4H4. Correct integration of either cassette was confirmed at each step by PCR analysis using primer pairs ATG9DETF and ATG9DETR, ATG9AMPR and ARG4DET2, or ATG9AMPF and HIS1F1268 (Table 2). Southern blot analysis was also performed using an ATG9-specific probe to the 3'-UTR of ATG9, amplified using primers ATG9PBF and ATG9PBR (Table 2). Correct gene deletion resulted in the replacement of $2858 \mathrm{bp}$ of the $2859 \mathrm{bp}$ ATG9 ORF, with either the $2822 \mathrm{nt}$ HIS1 or $2161 \mathrm{nt} A R G 4$ encoding cassettes. Finally a wild-type copy of ATG9 including $5^{\prime}$ and $3^{\prime}$ flanking sequences was introduced to the deletion strains on pLA2, to produce a prototrophic 'reconstituted' strain. Prototrophic deletion strains were produced by transforming the deletion strains with plasmid vector alone (pLUX). Either plasmid was digested with NheI prior to transformation to target integration into (and reconstitution of) the URA3 loci. The presence/absence of ATG9 in the prototrophic deletion/reconstituted strains was confirmed by amplification using the ATG9DETF/R primer pair.

Strains harbouring API-GFP fusions were derived from BWP17 (ATG9/ATG9), BAA1 and BAA4 (ATG9/atg9A), and BAA1H1 and BAA4H4 (atg9 $/ \operatorname{atg} 9 \Delta)$ using the PCR-based tagging approach described by Gerami-Nejad et al. (2001). Primers LAP4GFPF and LAP4GFPR (Table 2) were designed to amplify a GFP : : URA3 cassette from plasmid pGFPURA3 (Gerami-Nejad et al., 2001) with $78 \mathrm{bp}$ of homology on either end to direct integration at the $3^{\prime}$ end of the $C$. albicans LAP41 gene to form an in-frame LAP41-GFP fusion. The amplified cassette was transformed into the above strains; transformant colonies were selected, and screened for correct integration by PCR. Genomic DNA was prepared from transformants and PCR-amplified using primers LAP4DETR and URA3-5 (Table 2); a product of $470 \mathrm{bp}$ confirmed the expected integration event.

DNA manipulations. PCR was performed using standard reagents. Plasmids pGEMURA3, pGEMHIS1 and pRSARG $\Delta$ Spe (Wilson et al., 1999) were provided by Dr A. Mitchell (Columbia University). Plasmid pLUX (Ramon \& Fonzi, 2003), was provided by Dr W. Fonzi (Georgetown University). Plasmids pLA2 and pLA9 were made by amplifying the ATG9 ORF with $693 \mathrm{bp}$ of $5^{\prime}$-UTR and $214 \mathrm{nt} 3^{\prime}$-UTR from genomic DNA with ATG9AMPF and ATG9AMPR (Table 2) to incorporate BamHI sites. The resulting product was cloned into the BamHI site of pLUX.

Growth conditions. Strains were routinely grown on YPD (1\% yeast extract, $2 \%$ Bacto Peptone, $2 \%$ glucose) at $30{ }^{\circ} \mathrm{C}$, supplemented with uridine $\left(25 \mu \mathrm{g} \mathrm{ml}^{-1}\right)$ when necessary (Guthrie \& Fink, 1991). For growth curves, overnight cultures were subcultured to $20 \mathrm{ml}$ fresh YPD medium to $\mathrm{OD}_{600} 0.2$ and incubated at $30^{\circ} \mathrm{C}$ with

Table 1. Strains

\begin{tabular}{|c|c|c|}
\hline Strain & Relevant genotype & Source \\
\hline SC5314 & ATG9/ATG9 & \\
\hline YJB6284 & ATG9/ATG9 & Bensen et al. (2002) \\
\hline BWP17 & 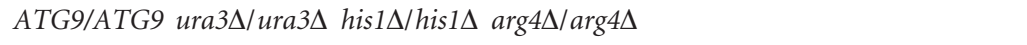 & Wilson et al. (1999) \\
\hline BAA1/4 & 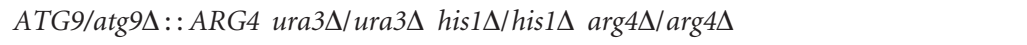 & This study \\
\hline BAA1H1/BAA4H4 & 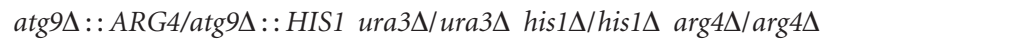 & This study \\
\hline AGD1 and AGD5 & 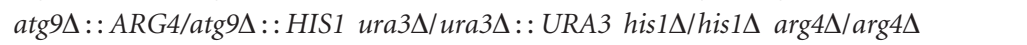 & This study \\
\hline AGR1 and AGR5 & 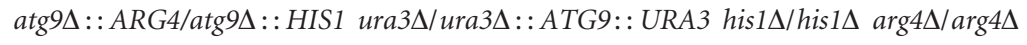 & This study \\
\hline $\mathrm{BL} 1 / 2$ & ATG9/ATG9 LAP4/LAP4-GFP & This study \\
\hline $\mathrm{LA} 1 / 2$ & ATG9/atg9D:: ARG4 LAP4/LAP4-GFP & This study \\
\hline DAL3/51 & $\operatorname{atg} 9 \Delta / \operatorname{atg} 9 \Delta:: A R G 4$ LAP4/LAP4-GFP & This study \\
\hline
\end{tabular}


Table 2. Oligonucleotides

\begin{tabular}{|c|c|}
\hline Primer & Sequence $\left(5^{\prime} \rightarrow 3^{\prime}\right)$ \\
\hline ATG9AMPR * & TCATCAGGATCCAATAGAAAATAAAATCAACCC \\
\hline ATG9AMPF* & TCATCAGGATCCACTTCTTTTGACGATCAACCC \\
\hline ATG9DISF & $\begin{array}{l}\text { TTCACTTATCCTACTTTTCTTCTTTTTGAAGAATAACACAAGCTAACTTGTTCA- } \\
\text { TGCTGGATGATAAGATTTGTGGAATTGTGAGCGGATA }\end{array}$ \\
\hline ATG9DISR & $\begin{array}{l}\text { CTCTCATTCCСТCCAAAAATAACATTATATAAACCAATGTATGTAAAACTAAC- } \\
\text { GTAGTAATACTAATTACCTTTCCCAGTCACGACGTT }\end{array}$ \\
\hline ATG9PBF & CGTTAGTTTTACATACATTGG \\
\hline ATG9PBR & AAGTATCACGATTAGTTCGAG \\
\hline ATG9DETF & TCATATATAATCAACAGGGGC \\
\hline ATG9DETR & AGTTATAGCCCAAATTGTCCC \\
\hline LAP4GFPF & $\begin{array}{l}\text { ATTAGGTATTAAATTCTTCTATGGTTTCTTCAAGAATTGGAGAGATGTCTATG- } \\
\text { ATAATTTTGTTGATTTAGGTGGTGGTTCTAAAGGTGAAGAATTATT }\end{array}$ \\
\hline LAP4GFPR & $\begin{array}{l}\text { TAAACTAACAAATAACTAATACTTGCAAATCAACTTGCAAATCAACTTTTAATG- } \\
\text { ATTCCTTTTCCTGTTGTTCAATGTTCGATCTAGAAGGACCACCTTTGATTG }\end{array}$ \\
\hline LAP4DETR & TTAATGTACAAGCCTTGCGGC \\
\hline ARG4DET2 & ATCAATTAACACAGAGATACC \\
\hline HIS1F1268 & CCGCTACTGTCTCTACTTTG \\
\hline URA3-5 & CCTATGAATCCACTATTGAACC \\
\hline
\end{tabular}

${ }^{\star}$ BamHI sites shown in italic.

shaking. $\mathrm{OD}_{600}$ was determined from samples taken hourly. Transformants were selected on minimal media $\left[6.75 \mathrm{~g} \mathrm{l}^{-1}\right.$ yeast nitrogen base plus ammonium sulfate and without amino acids, $2 \%$ glucose, 2\% Bacto agar (YNB)] supplemented with the appropriate auxotrophic requirements, as described for $S$. cerevisiae (Burke et al., 2000), except for uridine, which was added at $25 \mu \mathrm{g} \mathrm{ml}{ }^{-1}$.

Phenotypic assays. Resistance to temperature stress was determined on YPD agar at 37 and $42{ }^{\circ} \mathrm{C}$, and osmotic stress on YPD agar plus $2.5 \mathrm{M}$ glycerol or $1.5 \mathrm{M} \mathrm{NaCl}$. Secreted aspartyl protease (SAP) secretion was examined on BSA + YE agar (Crandall \& Edwards, 1987). Carboxypeptidase Y (CPY) activity was measured using a colorimetric assay as previously reported (Palmer et al., 2003). Resistance to nitrogen starvation was determined using an assay similar to that of Noda et al. (2000). Each strain was grown in YNB broth for $48 \mathrm{~h}$ at $30^{\circ} \mathrm{C}$. Cells were washed twice in SD-N and $10^{7}$ cells resuspended in $2 \mathrm{ml} \mathrm{SD-N}$ medium $(0.17 \%$ yeast nitrogen base without ammonium sulfate or amino acids, $2 \%$ glucose). Samples taken at intervals were plated to YPD agar, and viability determined as c.f.u. after 2 days at $30^{\circ} \mathrm{C}$. Accumulation of autophagic bodies within the vacuole was assayed by transferring cells grown overnight in YPD to SD-N medium in the presence of $1 \mathrm{mM}$ PMSF (Noda et al., 2000). PMSF inhibits the breakdown of the delivered autophagic bodies. After $6-24 \mathrm{~h}$ at $30^{\circ} \mathrm{C}$, cells were examined by differential interference contrast (DIC) microscopy. Filamentation on M199 and $10 \%$ fetal calf serum (FCS) agar was performed as previously described (Palmer \& Sturtevant, 2004). Cells from overnight cultures were also induced to filament in $10 \%$ FCS (in distilled $\mathrm{H}_{2} \mathrm{O}$ ) at $37^{\circ} \mathrm{C}$ after inoculation at $10^{6}$ cells $\mathrm{ml}^{-1}$. Chlamydospores were induced on cornstarch-Tween agar as previously described (Palmer et al., 2004). Sensitivity to $\mathrm{H}_{2} \mathrm{O}_{2}$ and rapamycin was determined by measuring growth in YPD medium supplemented with the appropriate compound. Approximately 1000 cells from an overnight culture were inoculated to $200 \mu \mathrm{l}$ growth medium within the wells of a 96-well plate. After $48 \mathrm{~h}$ incubation at $30{ }^{\circ} \mathrm{C}$ (250 r.p.m.), growth was measured at $\mathrm{OD}_{600}$ using a plate reader.
Phagocytic assays. The murine macrophage cell line, J774A.1 (ATCC TIB-67) was grown according to ATTC instructions in DMEM, high glucose, $4 \mathrm{mM}$ glutamine, $10 \%$ fetal bovine serum at $37^{\circ} \mathrm{C}$ under $5 \% \mathrm{CO}_{2}$. The same culture conditions were used for incubations with C. albicans. C. albicans strains were incubated with J774A.1 cells as described by Lorenz et al. (2004). Briefly, J774.A1 cells were seeded overnight in 12 -well plates $\left(2 \times 10^{5}\right.$ per well) on $18 \mathrm{~mm}$ coverslips and incubated at $37^{\circ} \mathrm{C}$ under $5 \% \mathrm{CO}_{2}$. C. albicans strains were grown overnight at $30^{\circ} \mathrm{C}$, washed and incubated with J774A.1 cells at an m.o.i. of $2.0\left(4 \times 10^{5}\right.$ per well $)$ unless otherwise noted.

Macrophage survival. C. albicans and J774A.1 cells were incubated for 1,5 and $24 \mathrm{~h}$ and then wells were washed and incubated with $0.2 \mu \mathrm{M}$ calcein AM (final concentration) (LIVE/DEAD Viability/ Cytotoxicity Kit, Molecular Probes). C. albicans cells were simultaneously stained with calcofluor $(0.225 \mu \mathrm{M})$. Coverslips were removed from wells and observed under a fluorescence microscope. Macrophages that fluoresced green were viable. Macrophage survival was quantified by counting at least four fields for each well. Results are presented as the mean number of macrophages observed per field.

C. albicans survival. This was assessed using an end-point dilution assay as described by Rocha et al. (2001). Macrophages were seeded overnight in 96 -well plates at $5 \times 10^{3}$ per well. C. albicans $(50 \mu \mathrm{l})$ were added to the first column of cells $(150 \mu \mathrm{l})$, then serially diluted $1: 4$ for six columns so that the resulting m.o.i. were between 2 and $1.9 \times 10^{-3}$. The plates were incubated for $48 \mathrm{~h}$. Controls were wells with C. albicans but no macrophages. The lowest dilution of the control well where it was possible to discriminate distinct colonies was counted. The same dilution was counted for the C. albicans plus macrophage well. Results are presented as number of colonies in the presence of macrophages/number of colonies in the absence of macrophages $) \times 100$. Each experiment was set up in quadruplicate and $P$ values determined using the unpaired Student's $t$-test. 
Vacuole morphology and API localization. Vacuole morphology was visualized using the fluorescent dye FM4-64, as reported (Palmer et al., 2003; Vida \& Emr, 1995). In order to localize the API-GFP fusion protein, cells were grown into the exponential phase in YPD medium, stained with FM4-64, washed twice in distilled water, and visualized using an Olympus BX51 fluorescence microscope. All cells were applied to polylysine-coated slides prior to viewing.

Western blot analysis. Western blot analysis was performed basically as described previously (Palmer \& Sturtevant, 2004). Cell extracts of $C$. albicans were prepared by lysis with glass beads in the presence of a protease inhibitor cocktail (Sigma). Lysates were microfuged at 13000 r.p.m. to yield supernatant and pellet fractions. The pellet was resuspended in $50 \mu \mathrm{l}$ SDS-PAGE sample buffer. Protein lysates (pellet and supernatant fractions) prepared from equivalent numbers of cells were loaded per lane in $12 \%$ SDS-PAGE gels. Gels were transferred to PROTRAN (Schleicher and Schuell) and blotted with a rabbit polyclonal anti-GFP antibody (Anaspec). Equivalent loading of SDS-PAGE gels was confirmed by staining with Coomassie blue, and membranes were stained with Ponceau Red (Sigma) following the manufacturer's directions. A horseradishperoxidase-conjugated goat anti-rabbit secondary antibody (Rockland) was used for detection with reagents supplied by Pierce.

\section{RESULTS}

\section{Cloning and deletion of C. albicans ATG9}

S. cerevisiae ATG9 (also known as CVT7 and APG9) encodes an integral membrane protein of 998 amino acids, which is required for autophagosome and cvt vesicle formation (Lang et al., 2000; Noda et al., 2000). Deletion of ATG9 results in complete abrogation of autophagy and cvt pathways, leading to a block in API and AMS delivery, sensitivity to nitrogen starvation and an inability to complete sporulation (Lang et al., 2000; Noda et al., 2000). BLAST searches to the $C$. albicans genome sequence database (www.candidagenome.org/) identified a single ATG9 orthologue, predicted to encode a protein of 952 amino acids, sharing $32 \%$ identity and $47.6 \%$ similarity with S. cerevisiae Atg9p. A Kyte-Doolittle plot of the predicted C. albicans Atg9p sequence suggests six to eight transmembrane domains, which is in good agreement with the five to eight predicted transmembrane domains of the S. cerevisiae protein (Lang et al., 2000; Noda et al., 2000). In order to investigate the importance of autophagy (and the related cvt pathway) in the pathogen C. albicans, we constructed a strain in which both ATG9 alleles were deleted. Correct genotype was checked by both PCR detection and Southern blot analysis. The deletion mutant was readily produced, indicating that this gene is not essential for viability. A wild-type copy of ATG9 including $5^{\prime}$ and $3^{\prime}$ flanking sequences was then reintroduced to the deletion strain to produce a 'reconstituted' strain.

The atg9 mutant had a growth rate comparable to wildtype and reconstituted strains in liquid YPD culture, and was not sensitive to osmotic, temperature or oxidative stresses (data not shown). Levels of vacuolar CPY activity were also determined to be comparable between mutant and control strains, suggesting that trafficking from the Golgi apparatus to the vacuole was not affected in the $\operatorname{atg} 9 \Delta$ mutant (data not shown), and the mutant strain was unaffected in SAP activity (data not shown). The mutant strain was not sensitive to the autophagy-inducing drug rapamycin, or the replication checkpoint inhibitor caffeine (data not shown). Vacuole morphology was analysed using the fluorescent dye FM4-64 (Vida \& Emr, 1995). The atg9 mutant was observed to have an intact vacuole morphology, similar to that of $A T G 9^{+}$control strains (data not shown).

\section{C. albicans atg9 $\Delta$ is defective in autophagy}

We examined resistance to nitrogen starvation by measuring viability (as c.f.u.) after transfer to medium lacking a nitrogen source (SD-N) (Fig. 1a). Wild-type and reconstituted strains underwent two to three further cell divisions after shifting to SD-N medium, and then maintained viability for the duration of the experiment (30 days). However, the $\operatorname{atg} 9 \Delta$ strain did not continue to divide after the shift to SD-N and c.f.u. steadily declined to 0 , clearly demonstrating that $\operatorname{atg} 9 \Delta$ cells are sensitive to nitrogen

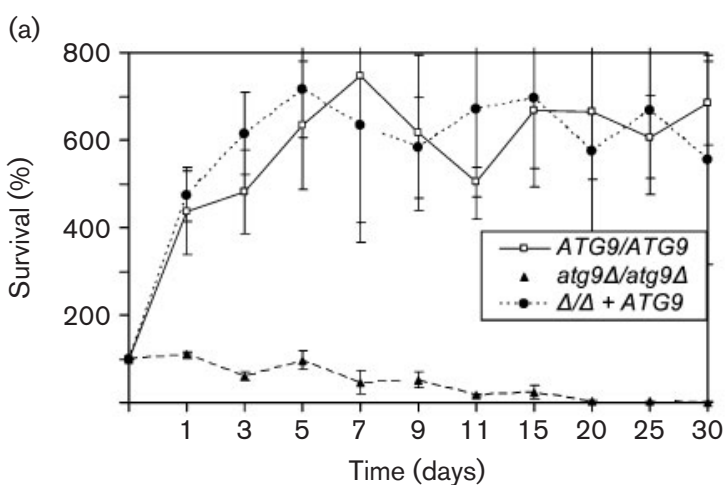

(b)

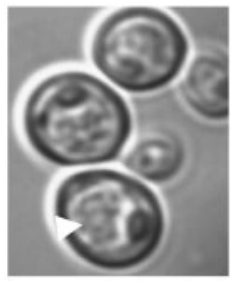

ATG9/ATG9

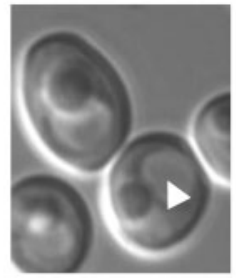

$\operatorname{atg} 9 \Delta / \operatorname{atg} 9 \Delta$

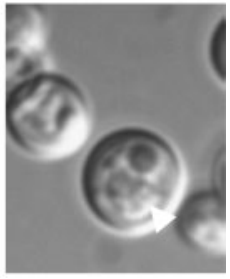

$\Delta / \Delta+A T G 9$
Fig. 1. C. albicans atg9 $9 \Delta$ mutant is defective in autophagy. (a) Resistance to nitrogen starvation was determined by measuring viability following shift to nitrogen-free (SD-N) medium. Viability was determined as c.f.u. and represented as a percentage of the c.f.u. at time 0. Data are given as the mean \pm SD of three experiments. (b) Autophagosome formation was induced in SD-N medium, supplemented with PMSF to inhibit the breakdown of the autophagic bodies following delivery to the vacuole. Cells were inspected by DIC microscopy after $16 \mathrm{~h}$ at $30{ }^{\circ} \mathrm{C}$. Arrowheads indicate vacuole containing accumulated autophagic bodies ( TTG9 $^{+}$strains), and vacuole lacking autophagic bodies (atg $9 \Delta / \operatorname{atg} 9 \Delta$ mutant). 
starvation. We next induced the formation of autophagosomes in SD-N media containing $1 \mathrm{mM}$ PMSF. PMSF is a serine protease inhibitor which blocks the action of vacuolar $\mathrm{PrB}$, an enzyme required for degradation of autophagic bodies within the vacuole (Noda et al., 2000). Under these conditions wild-type and ATG9 reconstituted strains accumulate autophagic bodies within the vacuole, giving the vacuole a granular appearance (Fig. 1b). As expected, the atg9 $9 \Delta$ strain did not accumulate autophagic bodies under these conditions, confirming that our atg $9 \Delta$ mutant is defective at an early step of autophagy, possibly autophagosome formation.

\section{C. albicans atg9 $\Delta$ is defective in cvt trafficking}

In order to establish if Atg9p functions in the cytoplasm to vacuole trafficking pathway, we tagged the cvt cargo protein API (encoded by the LAP4 gene in S. cerevisiae). Using the S. cerevisiae Lap4p sequence we identified two LAP4-like ORFs, designated LAP4 and LAP41 by the C. albicans genome database. The predicted amino acid sequences had $57 \%$ (Lap41p) and $34 \%$ (Lap4p) identity to S. cerevisiae Lap4p. We therefore selected the LAP41 ORF for GFP tagging using a PCR-based procedure (Gerami-Nejad et al., 2001), to generate a C-terminal Lap41-GFP fusion, in $A T G 9^{+}$and $\operatorname{atg} 9 \Delta$ backgrounds. Tagged strains were grown under nutrient-replete conditions (YPD) to the exponential phase of growth, conditions where cvt trafficking occurs but not autophagy, and observed by fluorescent microscopy (Fig. 2a). ATG $9^{+}$cells had fluorescence in three distinct patterns: 1 , single intense spot outside the vacuole; 2 , single intense spot within vacuole lumen; 3 , diffuse staining of the vacuole lumen. These patterns resemble API-GFP localization in S. cerevisiae (Suzuki et al., 2002) and correspond nicely with the cvt trafficking events: 1, oligomerized APIGFP precursor within the cytoplasm, or the membranebound cvt vesicle prior to vacuolar delivery; 2 , cvt body released into the vacuole; 3 , mature API-GFP released into the vacuole lumen following degradation of cvt vesicle (Wang \& Klionsky, 2003). The distribution of API-GFP in the atg $9 \Delta$ background mutant was altered (Table 3 ). No diffuse staining of the vacuole lumen was detected, and an increased proportion of cells had a single spot distribution. Furthermore, the spots were of increased intensity in the atg9 $9 \Delta$ mutant as compared to the $A T G 9^{+}$control strains, suggesting an accumulation of API-GFP in the oligomeric cytoplasmic cvt complex.

In S. cerevisiae, API is synthesized as an inactive precursor, which is activated upon delivery to the vacuole by proteolytic cleavage of an N-terminal propeptide of 45 amino acids (Oda et al., 1996). This can be followed as a shift in molecular mass on a Western blot (Klionsky et al., 1992; Suzuki et al., 2002). In order to further confirm the cvt trafficking defects, we immunodetected the API-GFP fusion protein in $A T G 9^{+}$and $\operatorname{atg} 9 \Delta$ backgrounds, using a polyclonal anti-GFP antibody. The predicted molecular mass of GFP is $26.9 \mathrm{kDa}$ and that of C. albicans Lap41p $56.7 \mathrm{kDa}$ (prior to proteolytic maturation). In $A T G 9^{+}$ (a)

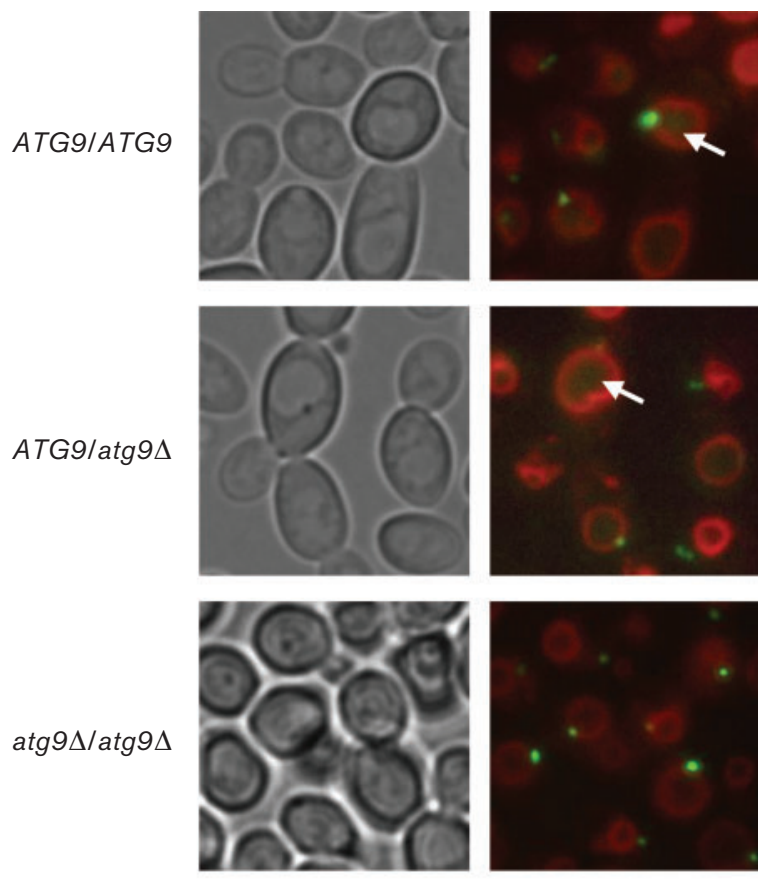

(b)

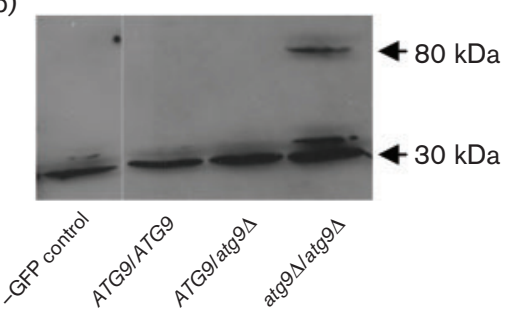

Fig. 2. C. albicans atg9 $\triangle 4$ mutant is defective in cvt trafficking. (a) The cvt cargo protein API (encoded by LAP41) was Cterminally GFP tagged in $\mathrm{ATG}^{+}$and atg9 9 genetic backgrounds. Cells were grown into the exponential phase and labelled with FM4-64 (red) to locate each cell's vacuole. Phase-contrast and GFP-FM4-64 combined images are shown for each strain. Arrows indicate faint diffuse GFP within the vacuole lumen $\left(\mathrm{ATGO}^{+}\right.$strains only). (b) API-GFP was detected using a polyclonal anti-GFP antibody. The antibody detected a non-specific band of around $25 \mathrm{kDa}$, and one of approximately $80 \mathrm{kDa}$ (atg9 $9 \Delta$ only), which corresponded with the expected molecular mass of Lap41-GFP. Two independently constructed GFP-tagged strains were analysed for each genotype, and both experiments repeated three times. Strain YJB6284 was used as the minus GFP control strain.

strains, delivery of API-GFP to the vacuole would expose the GFP tag to the proteolytically active vacuole lumen, which is liable to have resulted in its cleavage and/or degradation. This may account for the inability to detect the API-GFP fusion species by Western blotting (Fig. 2b), and the fairly low level of fluorescence detected within the vacuole lumen. Unfortunately the anti-GFP antibody recognized a peptide in the minus GFP negative control (lane 1, Fig. 2b), of 
Table 3. Vacuolar trafficking of API-GFP is dependent upon ATG9

The distribution of API-GFP was scored in $A T G 9^{+}$and atg9D genetic backgrounds. Cells were scored from three fields of view $(100 \times$ magnification $)$ for each of two independently constructed strains of each genotype. The proportion of cells in each GFP distribution category was calculated as a percentage of the total number of cells within the field. Data for all six fields for a given genotype were used to calculate the mean and standard deviation for each genotype. Results from a single experiment are shown. The experiment was repeated twice with similar results.

\begin{tabular}{|c|c|c|c|}
\hline \multirow[t]{2}{*}{ Genotype } & \multicolumn{3}{|c|}{ API-GFP distribution } \\
\hline & Vacuolar $^{*}$ & Point $\dagger$ & None $\ddagger$ \\
\hline ATG9/ATG9 & $71 \pm 1.4$ & $23.1 \pm 4.7$ & $5.9 \pm 4.8$ \\
\hline ATG9/atg9 & $65.7 \pm 6.2$ & $24.1 \pm 2.5$ & $10.3 \pm 3.7$ \\
\hline $\operatorname{atg} 9 \Delta / \operatorname{atg} 9 \Delta$ & $0 \pm 0 \S$ & $73.8 \pm 10.1 \S$ & $26.2 \pm 10.1 \S$ \\
\hline
\end{tabular}

${ }^{*}$ Vacuolar, diffuse GFP within vacuole lumen, with or without intense GFP spot in cytoplasm.

$†$ Point, intense GFP spot in cytoplasm, with no detectable staining of vacuole lumen.

$\ddagger$ None, no detectable GFP.

$\S P<0.05$.

around $25 \mathrm{kDa}$. A similar non-specific band has been described in S. cerevisiae (Suzuki et al., 2002) and may have obscured the detection of peptide species of a similar size, including any GFP that is cleaved upon vacuolar delivery. However, a species of $75-80 \mathrm{kDa}$, corresponding to the predicted API-GFP molecular mass was detected in the atg $9 \Delta$ background, indicating accumulation of API-GFP in the atg $9 \Delta$ mutant. This species was detected in the pelletable fraction of the cell extracts, but not the soluble fraction. This suggests that the API-GFP has accumulated as part of a large complex before vacuole delivery, perhaps either the highly oligomerized precursor API (cvt body), or even within a cvt vesicle (Yorimitsu \& Klionsky, 2005). These data clearly demonstrated that, similar to the $S$. cerevisiae atg $9 \Delta$ mutant, our C. albicans atg $9 \Delta$ mutant has a defect in cvt trafficking to the vacuole.

\section{Autophagy is not required for C. albicans differentiation}

In the model eukaryote $S$. cerevisiae, mutants defective in autophagy are unable to complete sporulation (Takeshige et al., 1992; Tsukada \& Ohsumi, 1993). Moreover, autophagy has been intimately associated with a wide range of differentiation and development events in higher eukaryotes. We therefore assessed the consequences of a defect in autophagy on two major differentiation processes of $C$. albicans. Yeast-hypha differentiation was examined on solid and in liquid M199 and FCS media. In each case the atg9 mutant produced filaments that were indistinguishable from the $A T G 9^{+}$control strains (data not shown), indicating that under in vitro conditions autophagy is not essential for yeast-hypha differentiation. Similarly, when induced on cornstarch-Tween agar, the atg9 $9 \Delta$ mutant produced chlamydospores with similar kinetics and abundance as the $A T G 9^{+}$control strains (data not shown). Together these results suggest that autophagy is not required for two major differentiation events in C. albicans.

\section{Autophagy is not required for C. albicans survival within or killing of a macrophage cell line}

Previous studies using two C. albicans mutants with defects in vacuole biogenesis demonstrated that some vacuolar function(s) are required for survival within, and killing of a mouse macrophage-like cell line (J774A.1) (Palmer et al., 2005). We considered that while not required for yeasthypha differentiation or chlamydospore formation, autophagy may be important for normal interaction with host cells or survival within host tissues. To test this we analysed the outcome of $\operatorname{atg} 9 \Delta$ interaction with the J774A.1 cell line, by determining macrophage and C. albicans survival rates. Following co-culture, the macrophages rapidly phagocytosed wild-type C. albicans. This was followed by filamentation of C. albicans within the macrophage, which resulted in stretching of the J774A.1 cells, followed by macrophage lysis. The atg9 mutant killed the J774A.1 cells as efficiently as $A T G 9^{+}$strains (data not shown), indicating that autophagy is not required for killing of the macrophage cell line. $C$. albicans survival of the macrophage challenge was determined using an end-point dilution assay (Rocha et al., 2001). We found that the atg9s cells exhibited similar susceptibility to the J774A.1 interaction as $A T G 9^{+}$control strains (data not shown), indicating that this pathway does not aid C. albicans survival within the J774A.1 cells.

\section{DISCUSSION}

Autophagy is a well-conserved pathway in eukaryotes, and serves to facilitate a diverse range of cellular activities. In this study we investigated the importance of autophagy and the closely related cvt pathway during differentiation and pathogenesis of the fungal pathogen C. albicans. We hypothesized that autophagy may be an important mechanism for survival and/or differentiation within host tissues. We considered that during adaptation to new host environments $C$. albicans may need to recycle endogenous macromolecules to provide nutrients until host sources can be tapped, or that in order to facilitate rapid differentiation within the host an 'elimination' of inappropriately expressed proteins may occur via autophagic degradation. We addressed these questions by analysing a mutant strain defective in autophagy. As expected, the atg $9 \Delta$ mutant strain constructed within this study was blocked in autophagy and the closely related cvt pathway, and was sensitive to nitrogen starvation. However, the atg $9 \Delta$ mutant had a normal growth rate, vacuolar CPY activity, resistance to osmotic and temperature stress, and vacuole morphology. These findings 
suggest that the atg9 $9 \Delta$ mutant does not have a generalized defect in vacuolar function (Palmer et al., 2005), but a specific defect in cvt/autophagy trafficking.

The atg9 $9 \Delta$ mutant has no detectable defect in either chlamydospore or yeast-hypha differentiation, a result which was surprising given the importance of this pathway in a diverse array of eukaryotic biological processes, including S. cerevisiae sporulation. Microarray studies by Lorenz et al. (2004) demonstrated that upon phagocytosis by J774A.1 cells, C. albicans undergoes a starvation-like transcriptional response, suggesting that the phagolysosome is a nutritionally poor environment. Vacuolar proteases including API, CPY and PrB are upregulated following macrophage ingestion, suggesting that vacuole-mediated proteolysis may be important for survival following phagocytosis. However, our atg9 $9 \Delta$ mutant was not deficient in its ability to survive within or kill a mouse macrophagelike cell line, suggesting that autophagy may not be required for C. albicans-macrophage interaction. This prompted us to conduct a small-scale pilot study to test the virulence of the atg9 mutant in the well-defined mouse model of haematogenously disseminated candidiasis. Only three mice were infected per C. albicans strain, but our results (mean survival time and fungal burden in the kidneys) clearly suggested that the atg $9 \Delta$ mutant is fully virulent (data not shown). Taken together, these results indicate that autophagy plays little or no role in C. albicans differentiation or pathogenesis. It remains possible that autophagy is important for the long-term survival of $C$. albicans as a commensal organism, or to reside on a peripheral host site such as skin, or perhaps within the environment.

Previously we found a C. albicans vps $11 \Delta$ mutant to be defective in vacuole biogenesis and to exhibit a range of pleiotropic phenotypes, including a reduced growth rate, sensitivity to a range of stresses and to nitrogen starvation, and reduced vacuolar and secreted protease activities (Palmer et al., 2003, 2005). Moreover, this mutant is unable to undergo yeast-hypha differentiation and was unable to kill the J774A.1 macrophage cell line. Work with a partially functional vps11 allele revealed that restoring a normal growth rate, stress resistance, and SAP and vacuolar hydrolase activities was not sufficient to restore normal filamentation or macrophage killing. Both vps11 mutants have highly fragmented vacuole morphology and are sensitive to nitrogen starvation, suggesting either of these vacuolar functions to be important in yeast-hypha differentiation and macrophage killing. As outlined above, the major role of the vacuole during nitrogen starvation is through the process of autophagy. The results presented here clearly demonstrate that vacuolar functions relating to autophagy are of little consequence in either yeast-hypha differentiation or interaction with macrophages.

Gow \& Gooday $(1982,1984)$ originally observed the vacuole to undergo a dynamic expansion during C. albicans germtube emergence from a parental yeast cell. Furthermore, during apical extension of the germ-tube, asymmetrical division of the protoplasm yields subapical compartments composed almost entirely of vacuole, whereas the protoplasm migrates at the hyphal tip (Barelle et al., 2003; Gow \& Gooday, 1984). After a delay, the highly vacuolated compartments regenerate cytoplasm and the vacuoles recede. This pattern of vacuole inheritance also seems to influence cell cycle progression and branching frequency of the hyphae (Barelle et al., 2003; Veses et al., 2005). At present the mechanism by which vacuole expansion is mediated, and its importance during host interaction, are unknown. Our results further strengthen the hypothesis that the major role the vacuole plays in yeast-hypha differentiation relates to its morphology, and perhaps its physical expansion to generate 'empty' hyphal compartments. Moreover, our results eliminate the autophagic and cvt trafficking pathways as mediating germ-tube induced vacuole expansion.

\section{ACKNOWLEDGEMENTS}

This work was supported by NIH grant R21AI60371, awarded to J. E. S. This publication was also made possible by grant number P20RR020160 from the National Center for Research Resources (NCRR), a component of the National Institutes of Health (NIH). Its contents are solely the responsibility of the authors and do not necessarily represent the official view of NCRR or NIH.

\section{REFERENCES}

Abeliovich, H. \& Klionsky, D. J. (2001). Autophagy in yeast: mechanistic insights and physiological function. Microbiol Mol Biol Rev 65, 463-479.

Barelle, C. J., Bohula, E. A., Kron, S. J., Wessels, D., Soll, D. R., Schafer, A., Brown, A. J. \& Gow, N. A. (2003). Asynchronous cell cycle and asymmetric vacuolar inheritance in true hyphae of Candida albicans. Eukaryot Cell 2, 398-410.

Bensen, E. S., Filler, S. G. \& Berman, J. (2002). A Forkhead transcription factor is important for true hyphal as well as yeast morphogenesis in Candida albicans. Eukaryotic Cell 1, 787-798.

Burke, D., Dawson, D. \& Stearns, T. (2000). Methods in Yeast Genetics: a Cold Spring Harbor Laboratory Course Manual. Cold Spring Harbor, NY: Cold Spring Harbor Laboratory.

Crandall, M. \& Edwards, J. E., Jr (1987). Segregation of proteinasenegative mutants from heterozygous Candida albicans. $J$ Gen Microbiol 133, 2817-2824.

de Repentigny, L., Lewandowski, D. \& Jolicoeur, P. (2004). Immunopathogenesis of oropharyngeal candidiasis in human immunodeficiency virus infection. Clin Microbiol Rev 17, 729-759.

Gerami-Nejad, M., Berman, J. \& Gale, C. A. (2001). Cassettes for PCR-mediated construction of green, yellow, and cyan fluorescent protein fusions in Candida albicans. Yeast 18, 859-864.

Gow, N. A. \& Gooday, G. W. (1982). Vacuolation, branch production and linear growth of germ tubes in Candida albicans. J Gen Microbiol 128, 2195-2198.

Gow, N. A. \& Gooday, G. W. (1984). A model for the germ tube formation and mycelial growth form of Candida albicans. Sabouraudia 22, 137-144.

Guthrie, C. \& Fink, G. (1991). Guide to Yeast Genetics and Molecular Biology. New York: Academic Press. 
Klionsky, D. J. (2005). The molecular machinery of autophagy: unanswered questions. J Cell Sci 118, 7-18.

Klionsky, D. J., Cueva, R. \& Yaver, D. S. (1992). Aminopeptidase I of Saccharomyces cerevisiae is localized to the vacuole independent of the secretory pathway. J Cell Biol 119, 287-299.

Kyte, J. \& Doolittle, R. F. (1982). A simple method for displaying the hydropathic character of a protein. J Mol Biol 157, 105-132.

Lang, T., Reiche, S., Straub, M., Bredschneider, M. \& Thumm, M. (2000). Autophagy and the cvt pathway both depend on AUT9. J Bacteriol 182, 2125-2133.

Lorenz, M. C., Bender, J. A. \& Fink, G. R. (2004). Transcriptional response of Candida albicans upon internalization by macrophages. Eukaryot Cell 3, 1076-1087.

Maertens, J., Vrebos, M. \& Boogaerts, M. (2001). Assessing risk factors for systemic fungal infections. Eur J Cancer Care 10, 56-62.

Mizushima, N. (2005). The pleiotropic role of autophagy: from protein metabolism to bactericide. Cell Death Differ 12 (Suppl. 2), $1535-1541$

Nair, U. \& Klionsky, D. J. (2005). Molecular mechanisms and regulation of specific and nonspecific autophagy pathways in yeast. J Biol Chem 280, 41785-41788.

Noda, T., Kim, J., Huang, W. P., Baba, M., Tokunaga, C., Ohsumi, Y. \& Klionsky, D. J. (2000). Apg9p/Cvt7p is an integral membrane protein required for transport vesicle formation in the Cvt and autophagy pathways. J Cell Biol 148, 465-480.

Oda, M. N., Scott, S. V., Hefner-Gravink, A., Caffarelli, A. D. \& Klionsky, D. J. (1996). Identification of a cytoplasm to vacuole targeting determinant in aminopeptidase I. J Cell Biol 132, 999-1010.

Palmer, G. E. \& Sturtevant, J. E. (2004). Random mutagenesis of an essential Candida albicans gene. Curr Genet 46, 343-356.

Palmer, G. E., Cashmore, A. \& Sturtevant, J. (2003). Candida albicans VPS11 is required for vacuole biogenesis and germ tube formation. Eukaryot Cell 2, 411-421.

Palmer, G. E., Johnson, K. J., Ghosh, S. \& Sturtevant, J. (2004). Mutant alleles of the essential 14-3-3 gene in Candida albicans distinguish between growth and filamentation. Microbiology 150, 1911-1924.
Palmer, G. E., Kelly, M. N. \& Sturtevant, J. E. (2005). The Candida albicans vacuole is required for differentiation and efficient macrophage killing. Eukaryot Cell 4, 1677-1686.

Ramon, A. M. \& Fonzi, W. A. (2003). Diverged binding specificity of Rim101p, the Candida albicans ortholog of PacC. Eukaryot Cell 2, 718-728.

Rocha, C. R., Schroppel, K., Harcus, D., Marcil, A., Dignard, D., Taylor, B. N., Thomas, D. Y., Whiteway, M. \& Leberer, E. (2001). Signaling through adenylyl cyclase is essential for hyphal growth and virulence in the pathogenic fungus Candida albicans. Mol Biol Cell 12, 3631-3643.

Suzuki, K., Kamada, Y. \& Ohsumi, Y. (2002). Studies of cargo delivery to the vacuole mediated by autophagosomes in Saccharomyces cerevisiae. Dev Cell 3, 815-824.

Takeshige, K., Baba, M., Tsuboi, S., Noda, T. \& Ohsumi, Y. (1992). Autophagy in yeast demonstrated with proteinase-deficient mutants and conditions for its induction. J Cell Biol 119, 301-311.

Thumm, M., Egner, R., Koch, B., Schlumpberger, M., Straub, M., Veenhuis, M. \& Wolf, D. H. (1994). Isolation of autophagocytosis mutants of Saccharomyces cerevisiae. FEBS Lett 349, 275-280.

Tsukada, M. \& Ohsumi, Y. (1993). Isolation and characterization of autophagy-defective mutants of Saccharomyces cerevisiae. FEBS Lett 333, 169-174.

Veses, V., Casanova, M., Murgui, A., Dominguez, A., Gow, N. A. \& Martinez, J. P. (2005). ABG1, a novel and essential Candida albicans gene encoding a vacuolar protein involved in cytokinesis and hyphal branching. Eukaryot Cell 4, 1088-1101.

Vida, T. A. \& Emr, S. D. (1995). A new vital stain for visualizing vacuolar membrane dynamics and endocytosis in yeast. J Cell Biol 128, 779-792.

Wang, C. W. \& Klionsky, D. J. (2003). The molecular mechanism of autophagy. Mol Med 9, 65-76.

Wilson, R. B., Davis, D. \& Mitchell, A. P. (1999). Rapid hypothesis testing with Candida albicans through gene disruption with short homology regions. J Bacteriol 181, 1868-1874.

Yorimitsu, T. \& Klionsky, D. J. (2005). Autophagy: molecular machinery for self-eating. Cell Death Differ 12 (Suppl. 2), 1542-1552.

Edited by: D Sanglard 